\title{
Detectability of micro-variables in the ASAS database
}

\author{
M. David ${ }^{1}$, H. Hensberge ${ }^{2}$, and C. Nitschelm ${ }^{3}$ \\ 1 Departement Wiskunde en Informatica, Universiteit Antwerpen, Middelheimlaan 1, 2020 Antwerpen, Belgium \\ e-mail: marc.david@ua.ac.be \\ 2 Royal Observatory of Belgium, Ringlaan 3, 1180 Brussels, Belgium \\ 3 Unidad de Astronomía, Facultad de Ciencias Básicas, Universidad de Antofagasta, Avenida Angamos 601, Antofagasta, Chile
}

Received 28 January 2013 / Accepted 19 July 2013

\begin{abstract}
Aims. We exploit nine years of photometry in the ASAS $V$-band catalogue to develop a method to select in an objective way the best quality data and detect low-amplitude variables. We concentrate on bright stars $(V<10)$ and avoid open cluster stellar densities. Methods. We use the (probable) members of the Sco-Cen associations as a test sample of bright stars that includes periodic early-type photometric variables as well as young late-type stars with cyclic or irregular variability patterns. We select grade-A observations with consistent photometry in all apertures, amounting to $88 \pm 4$ per cent of all grade-A data in most cases, and use the aperture with lowest dispersion of magnitudes (excluding one percent of data to avoid sparse surviving outliers) to search for periodicities, cycles and long-term trends. The well-known alias problems, due to the once-per-night observing cadence in the ASAS project, can often be alleviated for the bright stars by use of the HIPPARCos satellite photometry.

Results. Micro-variables with a range of variability as low as 0.01-0.02 mag for periodic variables ( $V$ magnitudes $7-10)$ and 0.04 mag for irregular long-term variables are detected. In total 37 light variables are identified: 14 were previously unknown, and another 12 were listed as suspected variables. The light variability in the young associations in Sco-Cen is briefly discussed.

Conclusions. The ASAS database contains much more information on photometric variables than retrieved commonly.
\end{abstract}

Key words. stars: variables: general - techniques: photometric - stars: individual: HD 46057 - stars: individual: HD 146285 stars: individual: HD 116794 - stars: individual: HD 119022

\section{Introduction}

The All Sky Automated Survey (ASAS, Pojmański 2001, 2002), is a project which photometrically monitors stars over a large part of the sky. It has been operational in the southern hemisphere, from the Las Campanas Observatory in Chile, since mid 2000 and in the northern hemisphere, from Haleakala on the Maui island of Hawaii, since mid 2006. A large number of new variables have been detected, catalogued and made available through the internet ${ }^{1}$. Many papers on specific kinds of light variables have been published. Almost all of them concentrate on larger amplitude variables, but efforts have been started to find lower-amplitude variables of particular types (Kiraga \& Stepien 2007; Pigulski \& Pojmański 2008). Nevertheless, there is general agreement on the fact that the database has not been fully exploited yet.

The aim of this paper is to describe a method of analysis that permits to increase the detection rate of micro-variables. This method was tested on a relatively small subsample of stars, allowing for detailed interactive evaluation of the analysis strategy. As a by-product we discuss shortly some items related to the observation and data reduction, that might contribute to a performance increase in the future.

The paper is structured as follows. After a short description of the ASAS project, the feasibility of detecting microvariables up to $V \approx 10$ is shown. Thereafter, the test sample (Sect. 4 ) is defined and the data-quality selection method is described (Sect. 5 to Sect. 7). A list of light variables and suspected light variables is presented in Sect. 8, and some data are presented in more

\footnotetext{
1 http://www. astrouw. edu.pl/asas
}

detail for illustrative purposes. Finally, general conclusions are given.

\section{Data}

We use ASAS $V$ filter data (exposures of $180 \mathrm{~s}$ ) from the southern survey. A $f / 2.8200 \mathrm{~mm}$ Minolta lens is used to image a field of $8.8^{\circ} \times 8.8^{\circ}$ on a $2048 \times 2048$ AP 10 Apogee detector. Useful data are available between the saturation limit $V=7$ (up to $V=8.5$ for few frames) and the detection threshold $V=14$. Magnitudes are catalogued for five different extraction apertures with diameters ranging from 2 to 6 pixels, corresponding to $30-90^{\prime \prime}$. Data for the same star obtained with different pointings for the centre of the frame are kept in different arrays, and may have offsets at the level of hundredths of magnitudes. This is due to the frame-based magnitude calibration procedure, since 2004 based on the Tycho catalogue without inclusion of colour terms. Each measurement is assigned a quality code (grade-A for the best data, up to grade-D), the majority being grade-A. In 2004, the data reduction was modified significantly. We refer to the data from either treatment as the old catalog and the new catalog, respectively. Notice that with each catalogued measurement, ASAS provides a kind of uncertainty estimate for each aperture, but this number refers to the frame as a whole rather than to individual measurements.

The choice of the optimal aperture depends on the magnitude of the object and on the presence of nearby sources. For clean sources, the contributions of the read-out noise and of bias in the background subtraction grow with aperture size, but too small apertures are sensitive to bias with the exact sub-pixel position 

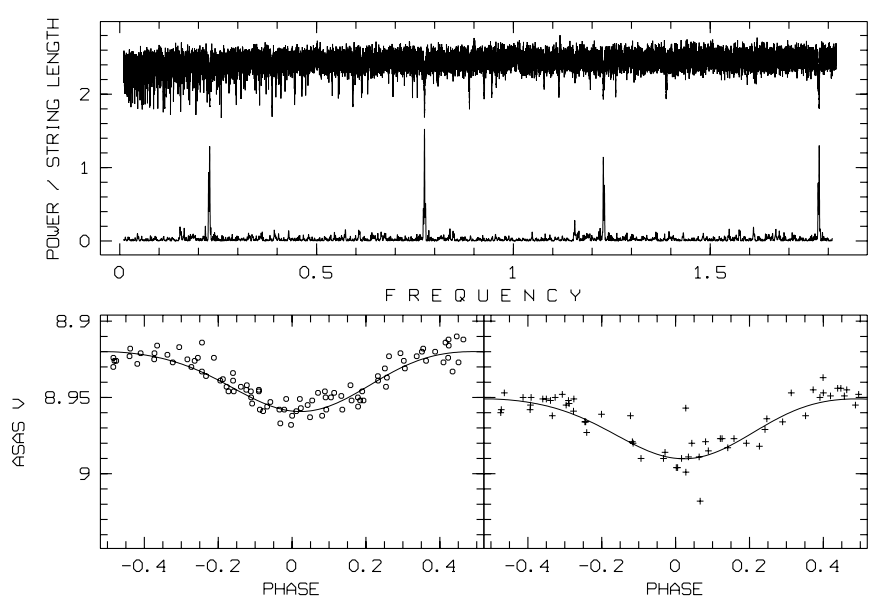

Fig. 1. Phase diagrams for the two old-catalogue data sets of HD 46057 using the ephemeris specified in Eq. (1) (lower panels) and the scargle and string-length statistic of the period search (upper panel). The string length is shifted upward by 1.5 to separate its minimum value from the maximum power of the scargle statistic.

of the centre of the object. For fainter stars, smaller apertures are expected to give a more precise result until a minimum size is reached at which discretisation bias dominates. However, for contaminated sources a smaller aperture than expected for its magnitude from the above considerations may be appropriate. The criterium adopted to select the optimal aperture is discussed in Sect. 5.

\section{Feasibility}

In order to assess the feasibility of using the ASAS photometry in a search for microvariables, we analysed data for a star with unknown, but expected microvariability, with magnitude $V=9$ (where the noise is not yet photon-limited) and in the field of an open cluster (i.e. a field with higher than average star density).

HD 46057 was detected as a B9p magnetic star (Hensberge et al. 1998) in the foreground of NGC 2244 (therefore also known as NGC 2244 \#381 as numbered by Ogura \& Ishida 1981). Such stars are microvariables, commonly with variability ranges of several hundredths of a magnitude in $V$. The light variability is due to the rotation of their inhomogeneous photosphere. It had not yet been the subject of a light variability analysis. Moreover, the ASAS data base contains two independent sets obtained from frames with a different central position: one set of 68 epochs and a second one of 160 epochs from frames nominally centered at the same right ascension (6h24) but at declinations differing nominally by 8 degrees. The numbers of observations refer to the old catalogue.

An analysis of the grade-A observations (61 and 92 epochs, respectively) gives convincing evidence for a periodic light variability within a range of $0.04 \mathrm{mag}$ (Fig. 1). The detection limit of periodic variability obviously lies at a significantly lower variability level. Data from frames with different centers are consistent except for a non-negligible shift in the mean level (about $0.03 \mathrm{mag}$ in this case), as clearly announced in the ASAS documentation. Notice the strong aliasing due to the quite strict one-day cycle observing mode.

With the newer data reduction, we found evidence of a significant increase in scatter. While the scatter around a smooth curve for high-quality old-catalogue data is $0.006 \mathrm{mag}$, it increases to $0.013 \mathrm{mag}$ for the high-quality new-catalogue data
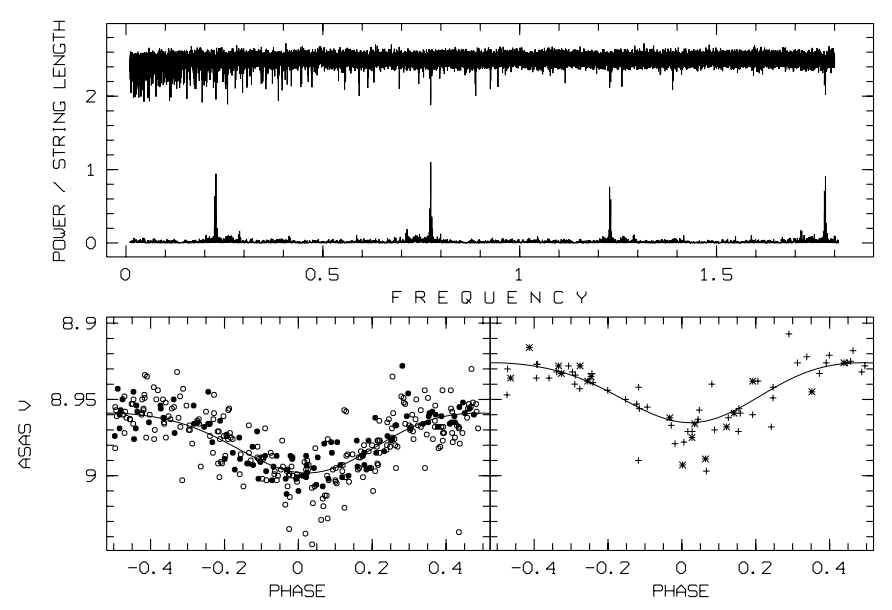

Fig. 2. Same as Fig. 1 but for the two new-catalogue data sets of HD 46057. The data corresponding to the same time interval as the old-catalogue data are shown as filled discs in the lower left panel and plusses in the lower right panel. Two substantially fainter grade-A data points are not shown on the scale of the lower left panel. Both statistics in the upper panel are "normalised" to remove their dependence on the sample size.

(Fig. 2), in agreement with the range of 0.010-0.015 mag found by Pigulski \& Pojmański (2008). If we restrict the comparison to the data obtained at the same epochs as in the old catalogue, the rms scatter is 0.010 mag for the corresponding new-catalogue data. The higher scatter is due almost entirely to a subset of $12 \%$ of "outliers" deviating by more than 0.015 mag from the model, while these are very rare in the corresponding old-catalogue data for HD 46057. Could the use of the Tycho catalogue (photometrically of low quality) for the zero-point determination of the fields in the new-catalogue have contributed to these offsets, for example in cases when the field centre was shifted sufficiently to alter the set of calibrator stars between exposures of (nominally) the same position?

Nevertheless, the much longer time baseline covered by the new-catalogue data improves the precision on the period by a factor 4. Finally, we notice that three of the four panels shown in both figures contain outliers (more often in the new catalogue). This motivated us to investigate on our whole test sample whether a more stringent selection criterion would be useful instead of just selecting grade-A data.

The light variability of HD 46057 is well described as

$\mathrm{HJD}($ min. light $)=2452939.85+(1.2917 \pm 0.0001) E$

$V_{\text {ASAS }}=8.936+0.0196 \cos (\phi-0.019)+0.0045 \cos (2 \phi-0.078)$

where the mean level refers to the larger of the old-catalogue sets and the small deviation from sinusoidality produces a broadening of the phase interval near maximum light. Since the shape of the light curve is nearly sinusoidal, a twice longer rotation period cannot be decisively rejected. Indeed, certain geometries may result in double-wave variations for a number of parameters (see e.g. Maitzen et al. 1978, for the case of HD 72968).

\section{Test sample}

We selected a test sample with the following characteristics: (a) containing periodic and cyclic photometric variables over a large range of spectral types; (b) covering a significant part of the sky (variation in time distribution of the observations); (c) large enough to reach statistically valid conclusions; (d) small enough 
to allow us to inspect the results in detail case per case; (e) containing stars for which photometry from another project is available. Especially the first two conditions suggest young, nearby stellar associations as interesting candidates. In the southern hemisphere, the Scorpius-Centaurus region is then the obvious choice.

In order to avoid field stars and to avoid objects which are too bright (with most ASAS images saturated), we select in this region the established members of the Sco-Cen complex, as defined by de Zeeuw et al. (1999), that are fainter than $V=7$. This leaves us with 344 objects. Requiring a minimum number of 40 grade-A observations per data set in the old ASAS catalogue, the test sample is reduced to 218 objects, represented by 235 data sets.

\section{Selection of extraction aperture}

\subsection{General case}

We use the aperture with which the magnitudes exhibited the lowest dispersion, with some precautions. Bearing in mind that the standard deviation of a time series of magnitudes for a given object is itself a random variable, we assessed the statistical significance of the dispersion-differences between different apertures by comparing them to the dispersion of these differences over an appropriate collection of data sets. In order to distinguish between statistics calculated over the selected exposures within a given data set from those calculated over the collection of data sets, we shall use subscripts exps and dss. Then we define

$$
v_{i}=\underset{\operatorname{exps}}{\operatorname{var}}\left[m_{i}\right], a_{i j}^{(v)}=\underset{\mathrm{dss}}{\operatorname{mean}}\left[v_{i}-v_{j}\right], \quad \sigma_{i j}^{(v)}=\sqrt{\frac{\operatorname{var}}{\mathrm{dss}}\left[v_{i}-v_{j}\right]}
$$

where $m_{i}$ represents the time-series of magnitudes obtained with aperture $\# i$. Thus we shall consider the difference between apertures $\# i$ and $\# j$ to be significant if

$\left|v_{i}-v_{j}-a_{i j}^{(v)}\right|>2.5 \sigma_{i j}^{(v)}$.

Table 1 summarizes the distribution of the "optimal" apertures in different bins reflecting the ASAS brightness of the object in the selected aperture. In view of our findings in Sect. 3, we evaluated these dispersions on the old-catalogue data (or rather what was left of it after severe damage caused by a RAID failure several years ago). In order to increase the sensitivity of the test, the time interval $1860 \leq \mathrm{HJD}-2.45 \times 10^{6}=\mathrm{RHJD} \leq 3300$ was used as earlier measurements contained for the brighter stars a larger amount of outliers (presumably due to a larger incidence of saturated central pixels).

First we note that, as one would expect, the smallest apertures \#0 and \#1 only rarely provide the smallest dispersion; in fact, most of the data sets where this occurs, afterwards turned out to be problematic in some sense. Clearly aperture \#3 appears to be preferred most frequently, but there is also a considerable number of cases where \#2 or \#4 provide the smallest dispersion. On the other hand, as the numbers in brackets indicate, in only few of these the difference with \#3 is statistically significant.

While this would suggest that, on the whole, the choice of an aperture is not critical, the increasing ratio of the frequencies \#2/\#4 shows that for weaker sources the preference is clearly shifted towards smaller apertures, for the reasons mentioned in
Table 1. Frequency with which the several extraction apertures provide the smallest magnitude-dispersion in different brightness classes.

\begin{tabular}{lccccc}
\hline \hline Brightness & $\# 0$ & $\# 1$ & $\# 2$ & $\# 3$ & $\# 4$ \\
\hline $7<m \leq 8$ & 1 & 0 & $9(0)$ & 38 & $13(1)$ \\
$8<m \leq 9$ & 0 & 1 & $23(1)$ & 54 & $29(2)$ \\
$9<m \leq 10$ & 0 & 0 & $25(1)$ & 24 & $9(1)$ \\
$10<m \leq 12$ & 1 & 1 & $7(0)$ & 0 & $0(0)$ \\
\hline
\end{tabular}

Notes. The numbers in brackets in Cols. \#2 and \#4 give the fraction for which the variance-difference with aperture \#3 satisfies Eq. (3).

Sect. 2. We conclude therefore that it is better to use the minimaldispersion aperture, even if its difference with an adjacent one does not seem significant.

\subsection{Inconsistent time series}

Even with the precautions already taken, still some of the time series are probably unreliable as a whole. Possible causes may be:

- one or more near-by objects contribute significantly to the signal we consider;

- the data set contains many exposures where the object in question was imaged close to the edge of the CCD (Pojmański 2002); unfortunately ASAS provides no information on this position so these cannot be eliminated a priori;

- the data set contains many exposures which suffered to some extent from saturation effects

but this list is by no means exhaustive. To identify potentially problematic data sets we looked for indications of inconsistency between the time-series obtained with successive apertures using the following approach. Let

$d_{i j}=\underset{\operatorname{exps}}{\operatorname{mean}}\left[m_{i}-m_{j}\right], \quad v_{i j}=\underset{\operatorname{exps}}{\operatorname{var}}\left[m_{i}-m_{j}\right]$.

In principle $d_{i j}$ and $v_{i j}$ should be dominated by random errors, in particular related to the (random) offset of the position of the source with respect to the centre of the detector bin; they should not depend on the potential variability of the observed objects. We shall assume that this is true for the majority of the data sets i.e., basically, that the magnitude difference $m_{i}-m_{j}$ is a normally distributed variable with a mean $\mu_{i j}$ and a dispersion $\sigma_{i j}$ which are common to all data sets which describe objects of comparable magnitude. Then we can use

$\mu_{i j} \approx \operatorname{mean}_{\mathrm{dss}}\left[d_{i j}\right], \quad \sigma_{i j}^{2} \approx \operatorname{mean}_{\mathrm{dss}}\left[v_{i j}\right]$

to state that

$z_{i j}=\frac{d_{i j}-\mu_{i j}}{\sigma_{i j}}$

has a standard normal distribution. Likewise, if $N$ represents the number of exposures in a given data set, then

$\chi_{i j}^{2}=\frac{(N-1) v_{i j}}{\sigma_{i j}^{2}}$ 
has a Chi-squared distribution with $N-1$ degrees of freedom. The statistics (6) and (7) can now be used to decide whether a given object exhibits an anomalous offset or variance between the apertures $\# i$ and $\# j$. This was used in particular to iterate the calculation of (5) so that they would not be biased by the outliers we aimed to detect.

For the final application, $z_{i j}$ and $\chi_{i j}^{2}$ may be combined in a single test. Since the sample mean and the sample variance (4) are statistically independent in our case, their joint distribution of $z_{i j}$ and $\chi_{i j}^{2}$ is the product of their marginal distributions. So the hypothesis that the magnitudes obtained with the apertures $\# i$ and $\# j$ are consistent, must be rejected at the significance level $\alpha$, if

$P_{i j}=\left(1-F\left(\chi_{i j}^{2}, N-1\right)\right)\left(1-\operatorname{erf}\left(\frac{\left|z_{i j}\right|}{\sqrt{2}}\right)\right)<\alpha$.

In order to avoid spurious effects due to the fact that the characteristics of the noise may change with the magnitude, the test was done separately for each 0.5 mag interval between 7 and 10 and for the region $m>10$, the latter being considered as a whole because a further subdivision would not leave enough objects in a group for the approximations (5) to be meaningful. Among the 235 datasets we found that 167 exhibited no inconsistencies at all, while in only three of them all aperture couples were inconsistent. In most of the remaining 65 datasets, only either the smallest or the largest apertures were problematic.

Depending on the origin of the inconsistency (if this can be guessed at all), either one or both of an inconsistent couple of time series must be considered as suspect. For instance, if the central pixel was saturated in many exposures this could lead to inconsistency in all couples involving the apertures \#0, \#1 and $\# 2$ while the couple (\#3,\#4) is consistent according to (8); then the latter does not necessarily imply that the time series $m_{4}$ is actually reliable. On the other hand, it seems reasonable to consider e.g. $m_{2}$ as reliable if only $m_{3}$ and $m_{4}$ are inconsistent due to the increasing influence of a near-by object. We should also remain aware of the fact that the condition (8) covers only a limited amount of information on the characteristics of the measurements so that it cannot be expected to classify these as good or bad in any overall sense.

Therefore the statistic $P_{i j}$ will not be used in the first place to exclude a number of objects from a detailed analysis but to explain (a posteriori) why a period search for a candidate variable (see Sect. 6) failed to give any definite result and, in some cases, to choose a different aperture than the one prescribed in Sect. 5.

\section{Selection of candidate variable stars}

In order to detect objects which are possibly variable we analysed the distribution of the standard deviations for our test sample and empirically derived the following magnitude-dependent criterion: a star is considered as a candidate variable when

$\sigma>1.8\left(0.005+0.001 \mathrm{e}^{m-8.5}\right)$

where $\sigma$ represents the standard deviation of the magnitudes in its time-series, for the aperture with minimum scatter, and $m$ the corresponding mean magnitude. The assumption that this criterion meaningfully distinguishes two kinds of objects is supported by the fact that the correlation between magnitudes obtained with successive apertures is significantly higher among the "candidate variables" than among the objects which do not satisfy (9), as illustrated in Fig. 3.
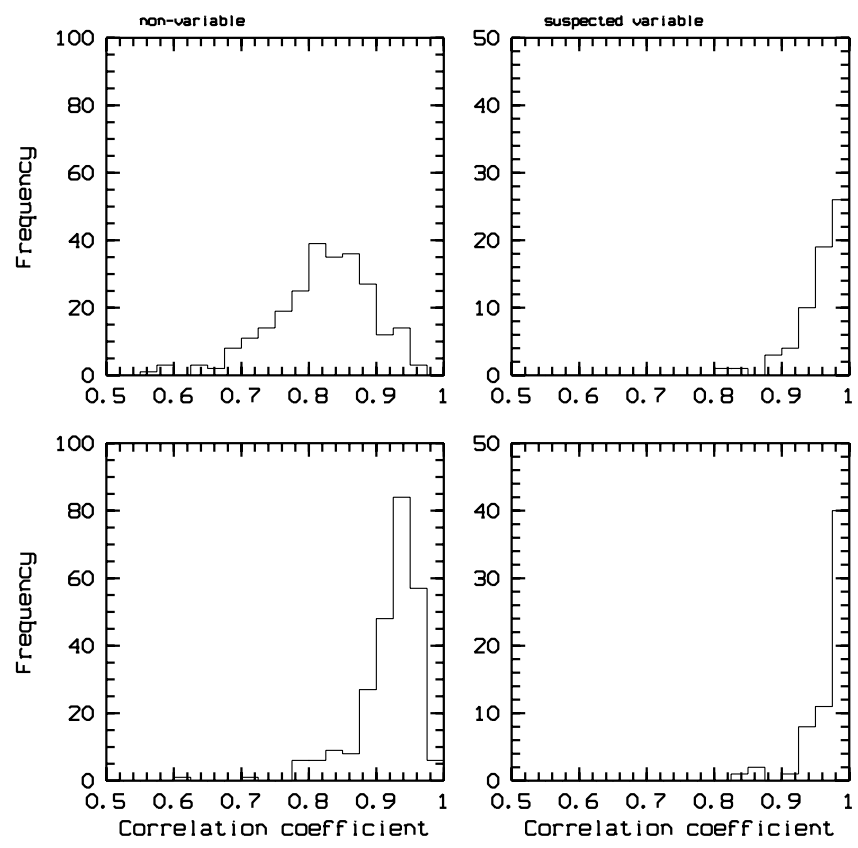

Fig. 3. Histogram of the correlation coefficients between the apertures \#2 and \#3 (upper row) and between \#3 and \#4 (lower row) in the "nonvariable" sample (left column) and in the "candidate variable" sample (right column) respectively.

Inevitably such a criterion may fail either way in marginal cases because of the statistical uncertainty on the standard deviations, in particular when the number of available exposures is low. False detections are not really a problem since they will simply fail the period-search afterwards. The risk of false rejections has been reduced by doing a period search anyway for some data sets which only marginally fail condition (9). However, variability amplitudes as low as the threshold are also at the limit of what was detectable as a periodic variation, so it is not surprising that these extra searches yielded no additional confirmed variables after all. Eventually 60 objects were identified as candidate variables and subjected to a detailed analysis.

For this sample of candidate-variables defined by the ASAS photometry, also the HIPPARCos photometry was checked. This allows us in some cases to choose between aliased frequencies, or to refine frequencies, or to extend the time baseline for long-term photometry. Following the advice given in the catalogue publication, measurements with identified reduction problems were not considered. Moreover, measurements with significantly higher estimated uncertainties in the catalogue were removed from the data set. Indeed, experience based on comparison of HIPPARCos photometry with precise ground-based photometry for micro-variables with well-known periods lead us to suspect that the higher uncertainty estimate is not a consequence of higher random noise, but rather an indication of bias in the measurement. Finally, at the magnitudes involved here, the HIPPARCos data have uncertainties on the level of 0.01 to $0.02 \mathrm{mag}$, which is a major drawback for the detection of micro-variables. With the specific observing sequence of the HIPPARCos satellite (two observations within twenty minutes, often repeated two hours later) the quality of the photometry at a specific epoch for longer-period micro-variables can be improved by averaging over consecutive observations within a small fraction, say one twentieth, of a period. This was applied in searches for longer periods in cases where evidence for shortterm variations was lacking. Indeed, a smaller amount of more 
accurate observations was found to provide a better constraint on the period.

\section{Optimising data sets for period search}

With the 60 candidate variable stars being identified, the period search was performed on the new catalogue data. Indeed, in spite of the argument given in Sect. 3, the sample size and time basis of the new catalogue data more than compensate the increased scatter in the new data reduction. In total, 108 data sets from the new catalogue were analysed. Whenever possible, the period search algorithm was applied on a combination of the different sets of a same object after defining a magnitude offset between the sets. In case of suspected long-tem variability, the offset was defined using the overlapping time interval. Only sets with 100 or more grade-A data were included.

The data with the chosen aperture $i$ for any of the candidate variables are now submitted to a consistency test to remove outliers which would degrade the power spectra. These outliers are eliminated using an indirect, empirical criterion and not by the mere fact that their magnitudes deviate from the median value of the data. For any of the stars, the final data set has at least 300 measurements, the median size is close to 500 and a few objects have slightly more than 1000 measurements.

\subsection{Removal of inconsistent individual data}

Any data set, even if it is not suspect according to the criterion (8), may yet contain a limited number of exposures for which the magnitudes from different apertures are inconsistent, for example due to bad pixels, cosmic rays or any cause disturbing drastically the object shape. We identify the pairs $(i, j)$ of such magnitudes by the criterion

$\left|m_{i}\left(t_{k}\right)-m_{j}\left(t_{k}\right)-d_{i j}\right|>2.5 \sqrt{v_{i j}}$

where $t_{k}$ indicates the instant of exposure. Empirically, we found that application of this criterion, iteratively computing $d_{i j}$ and $v_{i j}$ over the acceptable data, eliminates almost all outliers in magnitude, although the selection is not made directly on an outlying magnitude in the optimal aperture sample. We required that all couples $(i, j)$ with $i$ referring to the optimal aperture fulfill the consistency criterion at time $t_{k}$. This is illustrated in Fig. 4. Very few outliers apparently survive the rejection criteria, and often could be removed lowering the factor 2.5 in Eq. (10) slightly. However, we felt that the factor 2.5 defines a useful over-all cutoff criterion.

It is evident that data which are unbiased in the optimal aperture, but biased in a larger aperture will be rejected too. Nevertheless, the slight reduction by the sample size due to the rejection of some unbiased data is much less harmful than the inclusion of outliers in the period search algorithm.

Our requirement on the consistency of the differential magnitudes between different apertures rejects $12 \pm 4 \%$ of the grade-A measurements for objects fainter than 8th magnitude, and up to 25 per cent for objects brighter than 7.6 mag (Fig. 5). The additional rejections for brighter objects reflect the known saturation problems on the central pixel(s) of these stars, which mostly corrupted grade-A observations obtained before March 2002. The presence of a larger amount of these early observations has also a minor effect at fainter magnitudes in the sense that objects with less than $14 \%$ early observations scatter less around the average value of $12 \%$ of rejections (Fig. 5). Finally, a marginal statistical tendency of less rejections at fainter magnitudes (at a rate
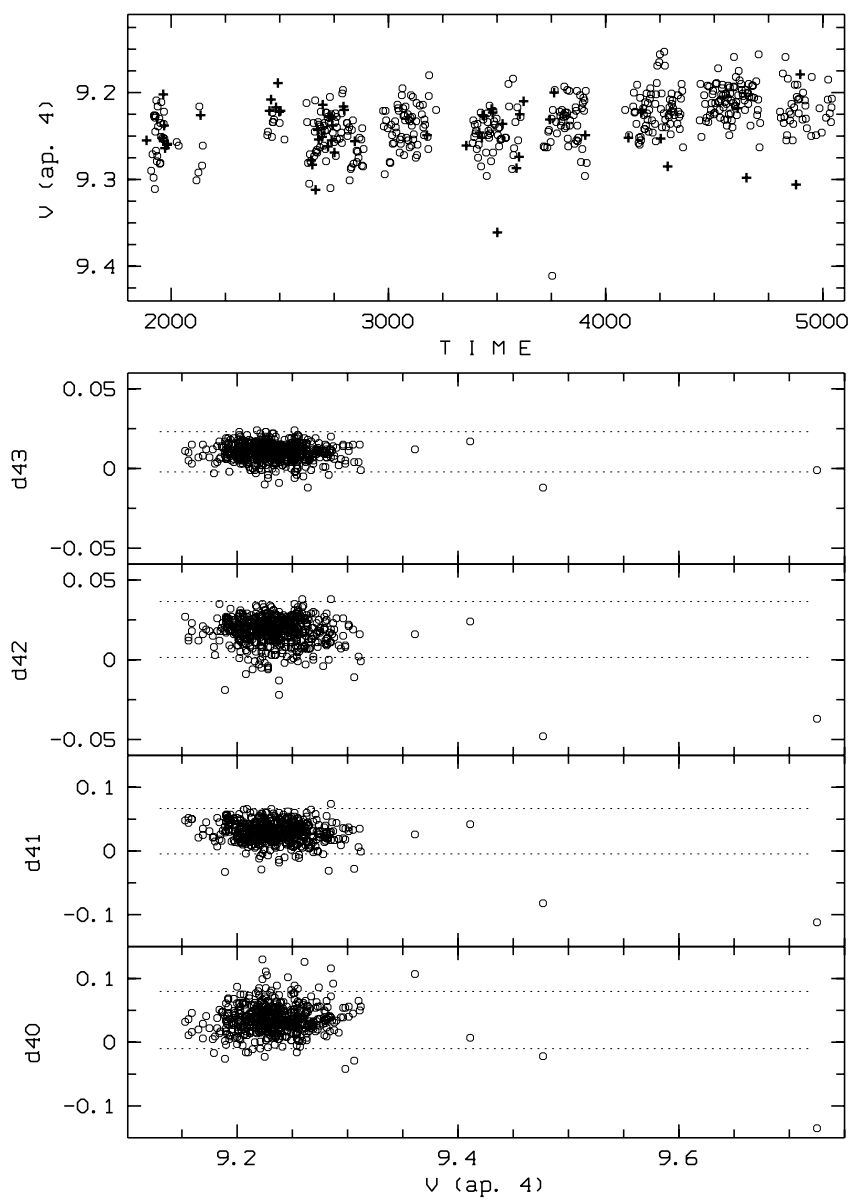

Fig. 4. Removal of data inconsistent in different aperture for HD 113466. The data outside of the dashed lines in the lower panels are rejected. The rejected data are shown by thick "+" in the upper panel (which does not show the faintest data point). Note that one obvious outlier in magnitude was not rejected, but is fairly close to the acceptance limit for $d_{43}$ and $d_{40}$.

of $1 \%$ per magnitude) can be understood as the consequence of increasing random scatter versus systematic bias towards fainter magnitudes. Typical cut-off values of $d_{4 j}$ are $0.010,0.018,0.036$ and $0.062 \mathrm{mag}$ for $j=3,2,1,0$ respectively, with values varying within $25 \%$ of these numbers for individual ASAS sets. Only $d_{43}$ and $d_{42}$ depend statistically slightly on magnitude, with typical values varying from 0.0095 and 0.017 at magnitude 7.5 to 0.0115 and 0.02 at magnitude 9.5 .

\subsection{Biased data sets}

Some objects show aperture-dependent photometry. With the large pixel size of the detector used by ASAS, nearby objects are often (partially) included in the extraction aperture. Figure 6 summarizes the situation. "+" symbols are used for objects showing a tendency to be brighter in larger apertures. Different sizes are used to indicate an increasing level of impact of the nearby objects. The largest-size symbols indicate that the aperture-dependent offset in magnitude is accompanied by a significant increase in the scatter of $m_{i}-m_{j}$ for the selected measurements. In these cases, the detection limit of the variability lies much higher than for isolated objects. Objects in this category turn out to be disturbed by objects which are 0.6 mag fainter to 1.5 mag brighter at distances between 30 and 65 arcsec. Still, larger-amplitude variability can be established 


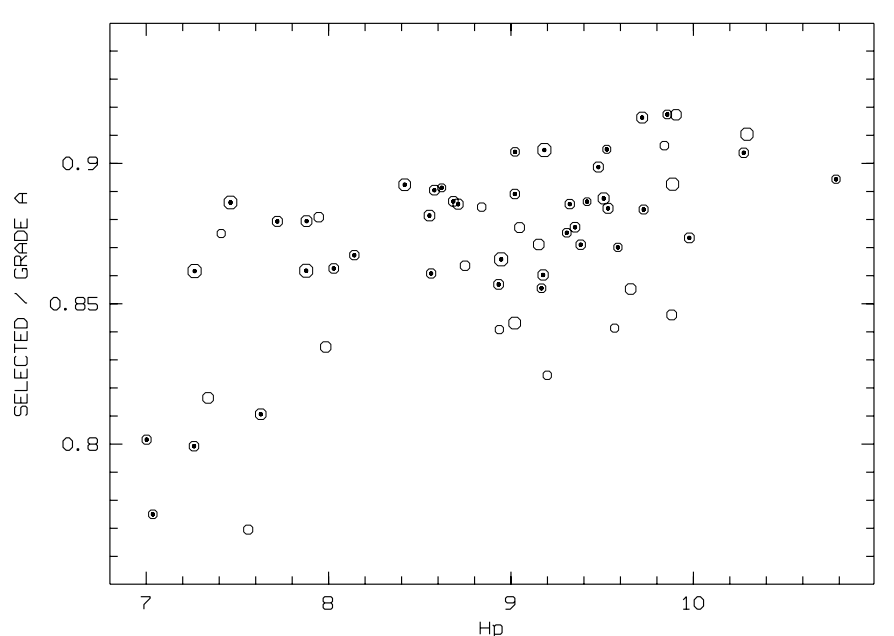

Fig. 5. Ratio of selected to all grade-A measurements as a function of HIPPARCOS magnitude $H_{\mathrm{p}}$. The area of the "o" symbols is proportional to the size of the grade-A samples (varying between 352 and 1048). Small dots in the symbols indicate the samples with less than $14 \%$ of the observations obtained before March 2002.

for the known chemically peculiar star HD $147010(\mathrm{Hp}=7.4)$ and for the known suspected variable HIP 68328 ( $\mathrm{Hp}=9.9)$. For the remaining three stars in this category, one can only state an upper limit of $0.05 \mathrm{mag}$ or less for the light variability, without excluding the possibility of non-variability (HD 111520, HD 111103 and HD 150418, see Table 4).

Medium-sized "+" symbols indicate a moderate to marginal increase in the scatter of the $m_{i}-m_{j}$ accompanying the magnitude offset between different apertures. Objects in this category turn out to be disturbed by stars which are either bright but relatively near to the main object (the case of HD 106444) or which are one to two magnitudes fainter and at distances of 36 to 52 arcsec (HD 133259, HD 103703, HD 108016). The efficiency of the light variability study is then only slightly reduced. Finally, the smaller "+" symbols correspond to cases with an aperture-dependent offset towards brighter magnitudes in larger apertures, but without noticeable influence on the scatter of the $m_{i}-m_{j}$ for the selected measurements. In those cases, the intervening stars are still fainter and the variability study is not affected.

In seven cases, one notices a tendency towards slightly fainter magnitudes in larger apertures, but without any effect on the scatter of the $m_{i}-m_{j}$. Those cases are represented in Fig. 6 by " $X$ " symbols. For few of them a component at 70-140 arcsec was identified that potentially has disturbed the background determination, but in other cases the presence of several fainter stars in this distance range could have contributed. The only noticeable influence on the variability study in those cases is the tendency that the lowest scatter in the data is obtained in apertures 2 and 3 rather than in the largest aperture.

The ASAS magnitudes used to construct the figure were derived from aperture 3 ("o" symbols) when the photometry was consistent in all apertures, and from aperture 1 otherwise. This removes largely the influence of components at distances well above 30 arcsec on the displayed ASAS magnitudes. The scatter around the mean relation displayed in Fig. 6 is dominated by the systematic uncertainties in the ASAS photometry. Two types of dashed lines indicate 2.5 times the rms scatter between ASAS and HIPPARCos magnitudes as given in Pojmański (2002), for stars near the centre and the edge of the frames, 0.045 and 0.0675

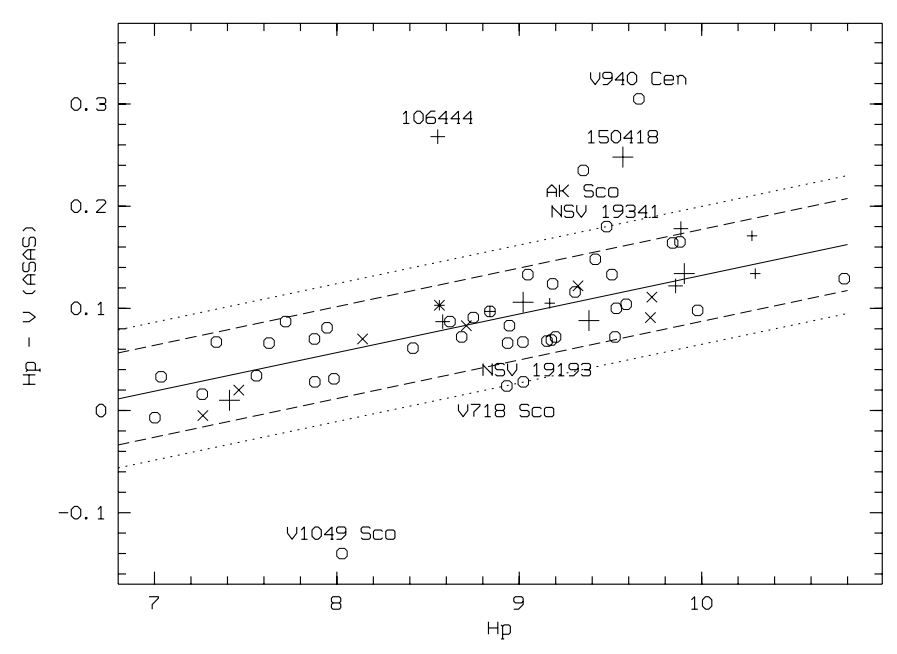

Fig. 6. Relation between HIPPARcos magnitude $H_{\mathrm{p}}$ and ASAS visual magnitudes derived from aperture 3 ("o") or aperture 1. See text for further explanation of symbols.

respectively. Inspecting the magnitudes in different ASAS subsets of the same star in our selection of candidate variable stars, we found differences of up to $0.04 \mathrm{mag}$.

The magnitude of a few stars differs more strongly. HD 106444 is a visual binary with the 0.8 mag fainter component at 24 arcsec, influencing the photometry in all apertures. HD 150418 has a 0.5 mag fainter companion at 31 arcsec distance (ASAS 164208-3130.1). In the case of V1049 Sco, AK Sco and V940 Cen there remains little doubt that their mean magnitude in the HIPPARCos era was significantly different from that in the ASAS era. Not surprisingly, it concerns two known highamplitude irregular variables and a M-type giant. For three already known or suspected variables which are near the 2.5 times rms limit at the edge of frames, there remains ambiguity about the interpretation.

\section{Variable stars and suspected variables}

The 60 selected objects include nine variables that previously received a variable star designation, one variable detected earlier in the framework of the ASAS project (Pojmański \& Maciejewski 2004), eleven stars designated as "new suspected variables" after the HIPPARCOS project and two variables detected afterwards by Koen \& Eyer (2002). All of these are confirmed in this work and for many of them we present new evidence for periods or cyclic variability. Our analysis identifies in addition fourteen previously unnoticed brightness variables, so that 37 of the 60 preselected objects end up as well-defined light variables.

An overview of these is presented in Table 2, where we indicate, in addition to quantitative information on the periods, cycle lengths and total range of variability, qualitatively the presence of different kinds of variability using the following symbols:

- I: irregular variable (on different time scales);

- P: periodic variable (only one period confirmed);

- mP: multi-periodic variable;

- qP: quasi-periodic variable (subsequent cycles not identical).

In some cases the distinction between $\mathrm{P}$ and $\mathrm{qP}$ is somewhat subjective. When more than one kind is identified in the same object, the dominant one is mentioned first (e.g. $\mathbf{P}+\mathbf{I}$ for a periodic variation superposed on an irregular variation which has a 
M. David et al.: Detectability of micro-variables in the ASAS database

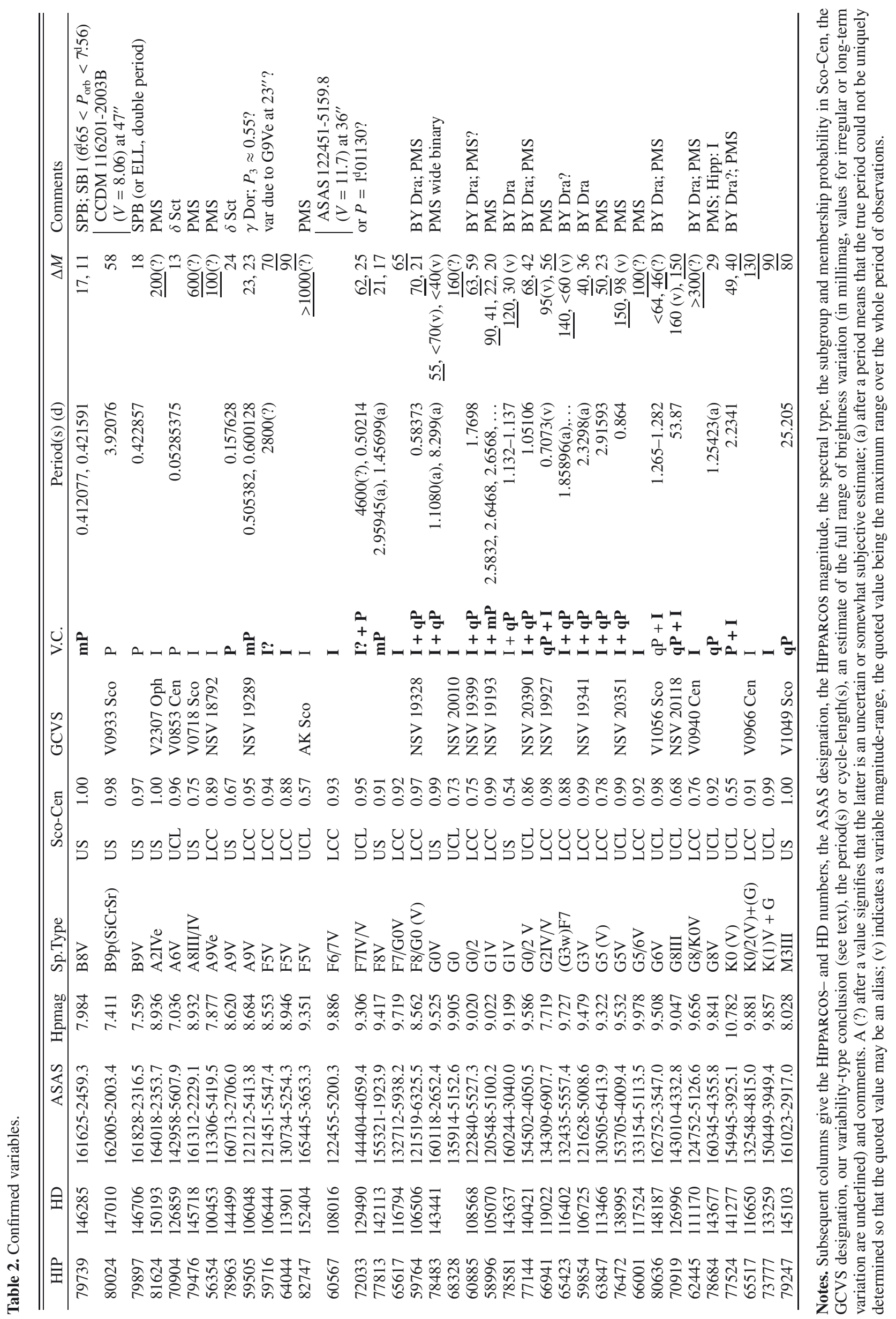


smaller amplitude). Boldface symbols indicate a new or substantially different result obtained during the present study. Periods and cycle lengths are arranged according to decreasing variability range.

Detailed analysis of a number of cool stars with larger amplitudes or obviously different scatter in yearly ASAS data sets illustrates the complexity of the variability, including slightly variable periods presumably related to differential rotation and spot location or migration, and largely varying amplitudes. Such analysis was not always performed for the lowest-amplitude variables although they may in principle show the same characteristics, but due to the lower amplitude at a less significant detection level.

The remaining twenty-three objects include stars we suspect to be variable ( 6 cases), and inconclusive cases, either because the inclusion in the candidate list might have been spurious (due to a small data set, a few outlying measurements, or a nearby star) or because a small amplitude of variability enhanced the scatter in a statistically detectable way, but no more decisive proof of variability can be retrieved. These objects are listed in Table 4 which furthermore gives the rms of their magnitude and comments on the plausibility of real variability or a possible cause of data corruption.

A detailed discussion of each of the variable stars will be presented in the Information Bulletin of Variable Stars, including a summary of the relevant literature and, for a minority of objects, comments on yet unpublished spectroscopy obtained by us. Here, we discuss only a few objects to illustrate the main types of detected variability (i.e. (multi)-periodicity, cyclic variability, long-term variability) and the detection limits set by the noise in the ASAS photometry and the analysis methodology.

\subsection{Example 1: low-amplitude multi-periodic variability}

HD $146285=$ HIP 79739, the hottest star in our list of variables, has spectral type B8V (Houk \& Smith-Moore 1988) and is a single-lined spectroscopic binary (Levato et al. 1987) with hitherto unknown orbital period. It is an example of the lowestamplitude periodic light variables detected in this study.

New high-resolution spectra obtained in three nights over a time baseline of eight days confirm the variable radial velocity, but the measured range in radial velocities $\left(10\right.$ to $\left.32 \mathrm{~km} \mathrm{~s}^{-1}\right)$ is quite different from the one presented by Levato et al. ( -41 to $15 \mathrm{~km} \mathrm{~s}^{-1}$ ). Both sets must therefore cover quite different parts of the orbital cycle. Combining the data (Table 3 ) reveals that the orbital period lies between 6.65 and 7.56 , but aliasing prevents a unique solution. Fitting orbits to 20 candidate periods selected from a period search algorithm reveals 13 possible, slightly eccentric, orbits which may represent the radial velocities with a rms scatter of $4.1 \mathrm{~km} \mathrm{~s}^{-1}$ or less $\left(2.6 \mathrm{~km} \mathrm{~s}^{-1} \mathrm{rms}\right.$ for the best solution). In view of the rms obtained by Levato et al. (1987) for similar systems, such a scatter is typical for their data. The present indeterminacy in the orbital period should be easily resolved by observations over a short time interval.

The present formally best solution is $P=7.21818, e=0.06$, $K_{1}=32.1 \mathrm{~km} \mathrm{~s}^{-1}, v_{\text {sys }}=-3.8 \mathrm{~km} \mathrm{~s}^{-1}, \omega=37 \mathrm{deg}$ implying a mass function $f(M)=0.025$ solar mass. The parameter range covered by the various solutions is $6.66<P<7$. $37, e<0.13$, $30.9<K_{1}<34.2$ and $0.021<f(M)<0.03$. In establishing these results we did not take into account the possibility of an offset in radial velocities between the two data sets, either of instrumental origin or because of a third body. We checked that reasonable values for such offset would improve the solutions only a little, although possibly extending the $K_{1}, e$ and
Table 3. Radial velocities of HD 146285.

\begin{tabular}{lrl}
\hline \hline HJD - 2 400 000 & $\begin{array}{r}\mathrm{RV} \\
\mathrm{km} \mathrm{s}^{-1}\end{array}$ & Source \\
\hline 42178.8250 & -41.1 & $\mathrm{~L}$ \\
42946.6560 & +14.6 & $\mathrm{~L}$ \\
43295.7020 & -0.2 & $\mathrm{~L}$ \\
43296.7420 & -23.2 & $\mathrm{~L}$ \\
43297.7160 & -32.8 & $\mathrm{~L}$ \\
43298.6250 & -23.6 & $\mathrm{~L}$ \\
43299.7760 & +2.1 & $\mathrm{~L}$ \\
55023.9698 & +29.7 & $\mathrm{D}$ \\
55023.9963 & +31.1 & $\mathrm{D}$ \\
55024.0497 & +31.8 & $\mathrm{D}$ \\
55024.1482 & +28.8 & $\mathrm{D}$ \\
55024.2085 & +28.0 & $\mathrm{D}$ \\
55031.1446 & +26.7 & $\mathrm{D}$ \\
55031.1853 & +26.5 & $\mathrm{D}$ \\
55032.0445 & +10.6 & $\mathrm{D}$ \\
55032.1085 & +9.8 & $\mathrm{D}$ \\
55032.2040 & +9.8 & $\mathrm{D}$ \\
\hline
\end{tabular}

Notes. The last column indicates the source, either L (Levato et al. 1987), or D (this paper).

$f(M)$ ranges to somewhat larger values. Figure 7 shows the data folded in the best orbital period and in one of the other candidate periods.

Rotation $\left(160 \mathrm{~km} \mathrm{~s}^{-1}\right)$ is not synchronized with the orbit, and the secondary is not detected by simple visual checks. It must be at least a factor eight fainter in the visual spectrum and the mass function together with the spectral type of the primary suggest it is heavier than 0.6 solar mass. No line profile variability is obvious. Both the HIPPARCos and the ASAS photometry exclude light variability with a frequency as low as the orbital one.

However, the ASAS photometry shows power at two frequencies around 2.4 cycles per day, and the HIPPARCOS photometry independently confirms the main frequency. Both amplitudes are between 5 and 10 millimag. In view of the relative faintness of the secondary component, both frequencies belong almost certainly to the primary. The spectral classification, our high-resolution spectra and the fast rotation are not characteristic for chemically peculiar magnetic stars. Hence, the primary is presumably a slowly-pulsating B star (SPB) with

$f_{1}=2.42673 \pm 0.00002$
$f_{2}=2.37196 \pm 0.00005$

The uncertainty on $f_{1}$ reflects the gain in precision by phasing together the ASAS and HIPPARCos data (Fig. 9). Few of these stars are known to have modes with as high a frequency as detected in this star (De Cat 2007).

Figure 9 shows that the microvariability appears more evidently in subsets with higher quality. We used different symbols to emphasize the HIPPARCos data which were obtained from two consecutive measurements within 20 min having both uncertainties of 11 millimag or below in the Epoch Photometry Annex of the HIPPARCos catalog. We also emphasized the ASAS photometry obtained in the time interval HJD 2452660 3300, which shows (for many stars) lower scatter. Apparently the data reduction and observation procedure were best fine-tuned to each other for this time interval.

Finally, it should be noticed that this is the only star in our sample for which a tail of faint data $(8.0<V<8.4)$, all obtained in 2001, was not well eliminated by our selection procedure 
Table 4. Doubtful candidate variables.

\begin{tabular}{|c|c|c|c|c|c|c|c|c|}
\hline HIP & HD & ASAS & Нp & Sp.Type & \multicolumn{2}{|c|}{ Sco-Cen } & rms & Comments \\
\hline 65021 & 115583 & $131943-6721.9$ & 7.265 & B9V & LCC & 0.81 & $13(2)$ & \\
\hline 73266 & 132094 & 145824-3721.7 & 7.262 & B9V & UCL & 0.85 & $15(4)$ & SB2?, see Sect. 3.2.3 \\
\hline 69845 & 124961 & $141739-4051.7$ & 7.879 & B9V & UCL & 0.96 & $13(4)$ & susp. var. $(P=29.36$ or 0.9671 ?) from best subset \\
\hline 78541 & 143488 & $160205-3644.6$ & 7.002 & $\mathrm{~A} 0 \mathrm{~V}$ & UCL & 0.45 & $14(3)$ & \\
\hline 73145 & 131835 & $145654-3541.7$ & 7.946 & A2IV & UCL & 0.99 & $13(4)$ & \\
\hline 66447 & 118379 & $133718-4053.9$ & 7.628 & A3IV/V & UCL & 1.00 & $12(4)$ & \\
\hline 60459 & 107821 & $122342-6352.2$ & 7.462 & A3 & LCC & 0.89 & $14(3)$ & \\
\hline 66908 & 119221 & $134244-4311.1$ & 7.339 & A4V & UCL & 0.99 & $11(4)$ & \\
\hline 56227 & 100282 & $113135-6743.9$ & 8.417 & FOIII & LCC & 0.88 & $11(2)$ & \\
\hline 63041 & 112109 & $125504-6338.4$ & 8.141 & F0V & LCC & 0.85 & $11(2)$ & \\
\hline 59084 & 105233 & $120701-5941.7$ & 8.710 & F0V & LCC & 0.54 & $12(2)$ & TYC $8644-954-1$ at $103^{\prime \prime}(V=11.4)$ \\
\hline 82218 & 151376 & $164747-1952.5$ & 9.152 & $\mathrm{~F} 2 / 3 \mathrm{~V}$ & US & 0.91 & $12(3)$ & if var., probably low ampl., high freq. (16-23 cy/day) \\
\hline 78233 & 142989 & $155829-2124.1$ & 9.167 & $\mathrm{~F} 2 / 3 \mathrm{IV} / \mathrm{V}$ & US & 1.00 & $13(3)$ & ASAS $f=13.082 \mathrm{cy} /$ day? not confirmed by HIPPARCOS \\
\hline 58220 & 103703 & $115627-5849.3$ & 8.580 & F3V & LCC & 0.93 & $21(2)$ & TYC $8643-1242-1$ at $42^{\prime \prime}(V=10.6)$ \\
\hline 64316 & & $131056-5147.1$ & 10.294 & F5 & $\mathrm{LCC}$ & 0.90 & $17(3)$ & \\
\hline 62657 & 111520 & $125020-4951.8$ & 9.021 & $\mathrm{~F} 5 / 6 \mathrm{~V}$ & $\mathrm{LCC}$ & 0.99 & $24(1)$ & CD-49 7416 at $49^{\prime \prime}(V=9.0)$ \\
\hline 79908 & 146610 & 161839-3839.2 & 9.176 & F7V & UCL & 0.78 & $20(3)$ & susp. long-term var. (35 mmag over 8 years) \\
\hline 62427 & 111103 & $124739-5824.9$ & 9.382 & F8 & LCC & 0.78 & $21(2)$ & CCDMJ12477-5826AB at $64^{\prime \prime}(V=8.0)$ \\
\hline 80535 & 148040 & $162630-2741.3$ & 8.748 & G0V & US & 1.00 & $18(4)$ & susp. long-term var. (30 mmag over 6.5 years) \\
\hline 61241 & & $123259-5106.8$ & 10.276 & G0 & LCC & 0.85 & $21(2)$ & \begin{tabular}{|l} 
susp. long-term var., range $\approx 27 \mathrm{mmag}$ ? \\
ASAS $123256-5107.0$ at $28^{\prime \prime}(V=12.1:)$
\end{tabular} \\
\hline 81775 & 150418 & $164210-3130.2$ & 9.568 & G5V & UCL & 0.68 & $25(4)$ & ASAS $164208-3130.1$ at $31^{\prime \prime} V=9.8$ \\
\hline 60913 & 108611 & $122902-6455.0$ & 9.183 & G5V & LCC & 0.97 & $16(3)$ & $\begin{array}{l}\text { susp. var. }(\text { range }<13 \text { ) } \\
P=625 \mathrm{~d} ? \text { and } P=13^{\mathrm{d}} .1 \text { or } 0 \mathrm{~d} 77 ?\end{array}$ \\
\hline 73783 & 133336 & $150452-3116.7$ & 8.839 & MOIII & UCL & 0.95 & $17(4)$ & susp. var., range too small to determine $P$ uniquely \\
\hline
\end{tabular}

Notes. Subsequent columns give the HIPPARCos - and HD numbers, the ASAS designation, the HIPPARCos magnitude, the spectral type, the subgroup and membership probability in Sco-Cen, the rms scatter for the ASAS set(s) in millimag with, between brackets, the aperture to which the scatter refers, and comments.

based on consistent photometry in all apertures. Thirty-six anomalously faint data remained in the final selection (one third of them near the rejection limit applied to $d_{40}$ ) of 87 data, while the number of outliers in the data obtained after 2001 is compatible with what we obtain for other stars (9 in 463). We assume that this is due to a non-identified technical problem and omitted these data in the above analysis.

\subsection{Example 2: Irregular long-term variability}

HD 116794 is a late-F star showing low-level long-term variability (Fig. 10). Apparently, maximum brightness occurs quite regularly about every 900 days. A search for shorter cycles or periods was performed after detrending the ASAS data with the spline fit shown in the figure. Power was found near $f=2.95 \mathrm{cy}-$ cles per day and near the aliases $f-1$ and $f+1$, but only at a level that we associated in other cases with a classification as suspected variable.

Confirmation was sought in the HIPPARCos data set; this spans a very large range in uncertainties and some short time windows with many observations: 85 of 212 data points are taken within 6 days centered on HJD 2448765.1 and 24 others within 3.3 days centered on HJD 2448 220.6. In other words, half of the HIPPARCos data set was obtained in these two short time intervals. These subsets should be insensitive to the longterm trends observed with ASAS. Unfortunately, while 22 of the 24 measurements in the latter subset have relatively low uncertainties of 0.024 mag or less, only 22 of the 85 measurements in the subset covering one week have this precision.

Figure 11 shows the scargle power diagrams obtained for the whole HIPPARCos sample with uncertainties of 0.024 mag or less, and for its two short subsamples. The HIPPARCos data do not support the frequency $f$. However, the short subsets respond both to frequencies near $f-1$. We conclude that a cycle with a period slightly above 0.5 might be present, but requires confirmation from adequately spaced and higher precision observations. Therefore, it is not mentioned in Table 2 . Using $v \sin i=126 \pm 9 \mathrm{~km} \mathrm{~s}^{-1}$ (Chen et al. 2011) implies $R \sin i=1.3$ solar radii. Hence, the suggested cycle length would be compatible with rotationally-induced variability. The estimated total range of variability is not larger than $0.015 \mathrm{mag}$.

\subsection{Example 3: Cyclic variability}

HD 119022 is a double star first resolved by HiPPARCos ( $\rho=$ 0.202 arcsec, $\Delta m=0.1 \pm 0.19)$ and in 1996 observed by Mason et al. (1998) $(\rho=0.103 \operatorname{arcsec}, \Delta m=0.7)$. While it has beyond doubt a fast-rotating component, the $v \sin i$ values reported in the literature diverge $\left(120 \pm 25 \mathrm{~km} \mathrm{~s}^{-1}\right.$, Soderblom et al. (1998); $215 \mathrm{~km} \mathrm{~s}^{-1}$, Torres et al. (2006); $145 \pm 9 \mathrm{~km} \mathrm{~s}^{-1}$, Chen et al. (2011). Henry et al. (1996) measure a high level of $\mathrm{Ca} \mathrm{H}$ and $\mathrm{K}$ emission, but $\mathrm{H} \alpha$ is strongly in absorption (Soderblom et al. 1998). The latter paper and Torres et al. (2006) also measure $\mathrm{Li}$ I equivalent widths of 0.35 and $0.2 \AA$, respectively, compatible with those measured by Xing (2010) in weak-line TTauri stars with similar spectral type and rotation period. We wonder whether Soderbloms remark that the star is somewhat enigmatic because its position is well-above the main-sequence (while the $\mathrm{H} \alpha$ absorption excludes it is a classical T Tauri type star), might be due to a luminosity estimate not taking into account the binary nature of the object. Spectral type estimates range from G0.5IV (Mamajek et al. 2002) to G5IV (Torres et al. 2006). The HIPPARCos catalogue identifies HD 119022 as a 

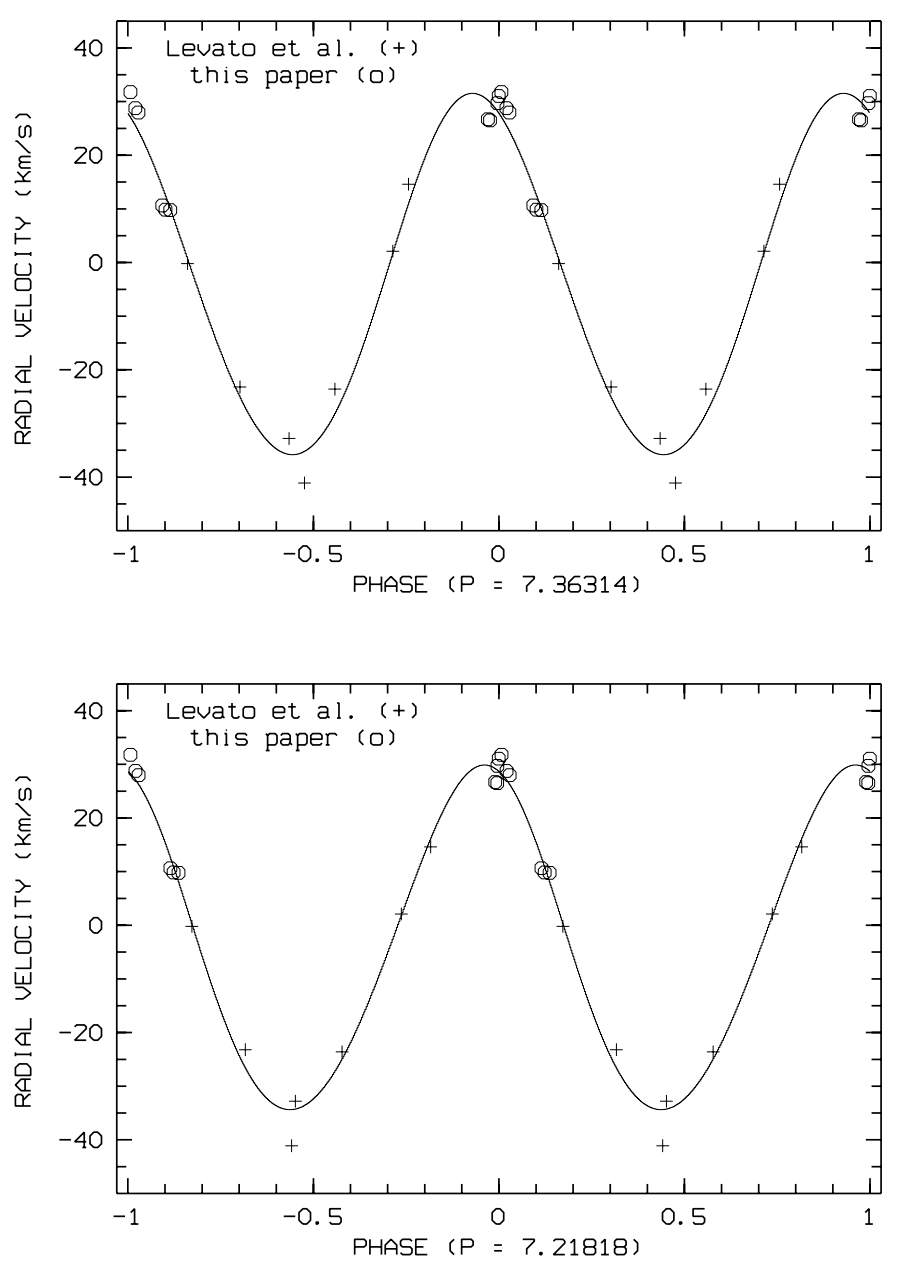

Fig. 7. Radial velocity data and some possible orbits for HD 146285.

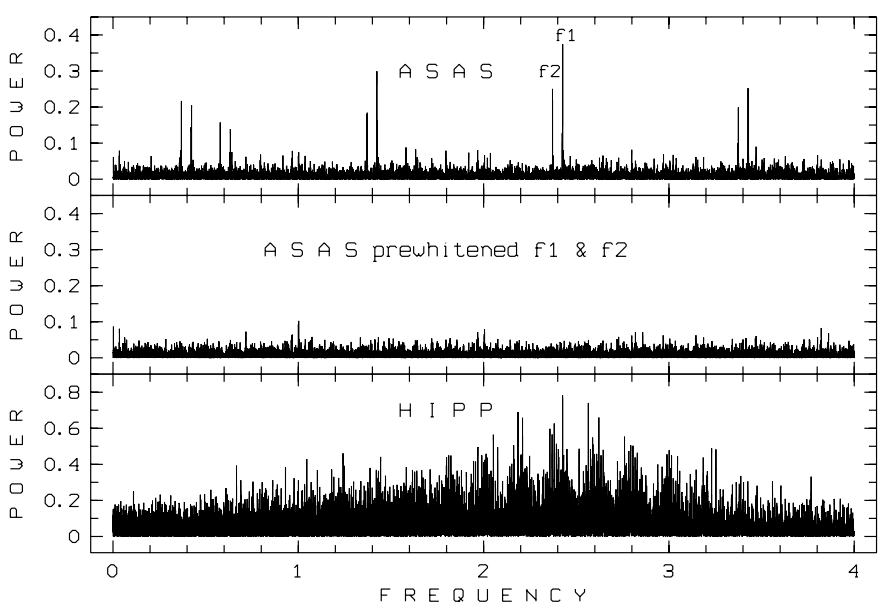

Fig. 8. Scargle power analysis of HD 146285: ASAS data (upper panel), the same data prewhitened for the frequencies $f_{1}$ and $f_{2}$ (middle) and HIPPARCOS data with uncertainties up to $0.016 \mathrm{mag}$.

light variable, and Koen \& Eyer (2002) derived a periodicity of $f=1.41015$ cycles per day with a low amplitude of $0.022 \mathrm{mag}$.

The ASAS photometry shows a moderate level of irregular long-term variations. It also confirms a short-term cycle with a frequency close to the one proposed by Koen \& Eyer (2002). At first sight, a scargle power diagram suggested multiple frequencies. Analysis of yearly subsets suggest a same cycle length in

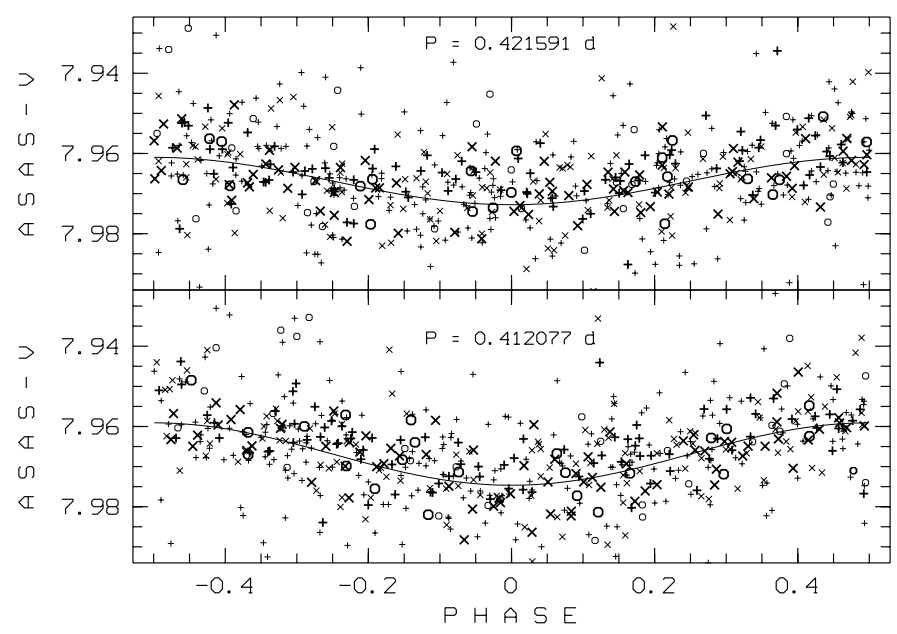

Fig. 9. Light-curves of HD 146285. HiPPARCos data (“o”) were shifted by -0.018 mag. Two ASAS subsets were used ("+" representing the larger one, " $\times$ " the smaller one). Larger, thicker symbols correspond to data believed to be statistically more precise (see text).
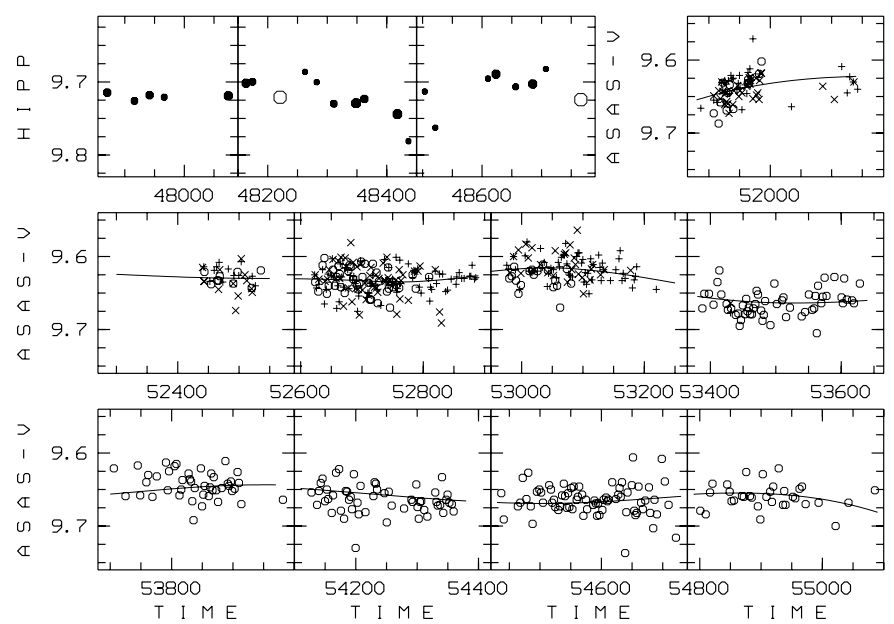

Fig. 10. Long-term light variability of HD 116794, from HIPPARCos and ASAS aperture-3 photometry. A smoothed spline for long-term detrending is shown in the ASAS panels. The data sets represented by "+" and " $\times$ " were shifted to 0.02 mag brighter magnitudes. Four ASAS measurements fainter than $V=9.76$ (out of 657 data) fall outside the scale of the figure. HIPPARCOS data are represented by dots of different sizes depending on the number of observations $(1,2-3,4-6)$ obtained within one day. Open circles refer to the two denser subsets discussed in the text.

most years (Fig. 13), but with largely different amplitude from year to year and with some phase shifts between subsequent years. This could be due to migration of and dynamical changes in stellar spots over time intervals shorter than for most late-type stars discussed in this work. A variability range of $0.095 \mathrm{mag}$ was attained during 2005, while it does not exceed 0.05 mag in any other year and stays below 0.03 mag in 2006 (Fig. 14). During 2003, 2006 and 2009 the frequency is very slightly, but significantly, different (in the two cases with most data much nearer to the $0.3 \%$ lower frequency found in the HIPPARCos photometry).

A closer look at the HIPPARcos photometry, especially the subset with uncertainties up to 0.013 mag, reveals that most power is due to the data after HJD 2448 150, suggesting again long-term variations in amplitude and phase. However, 


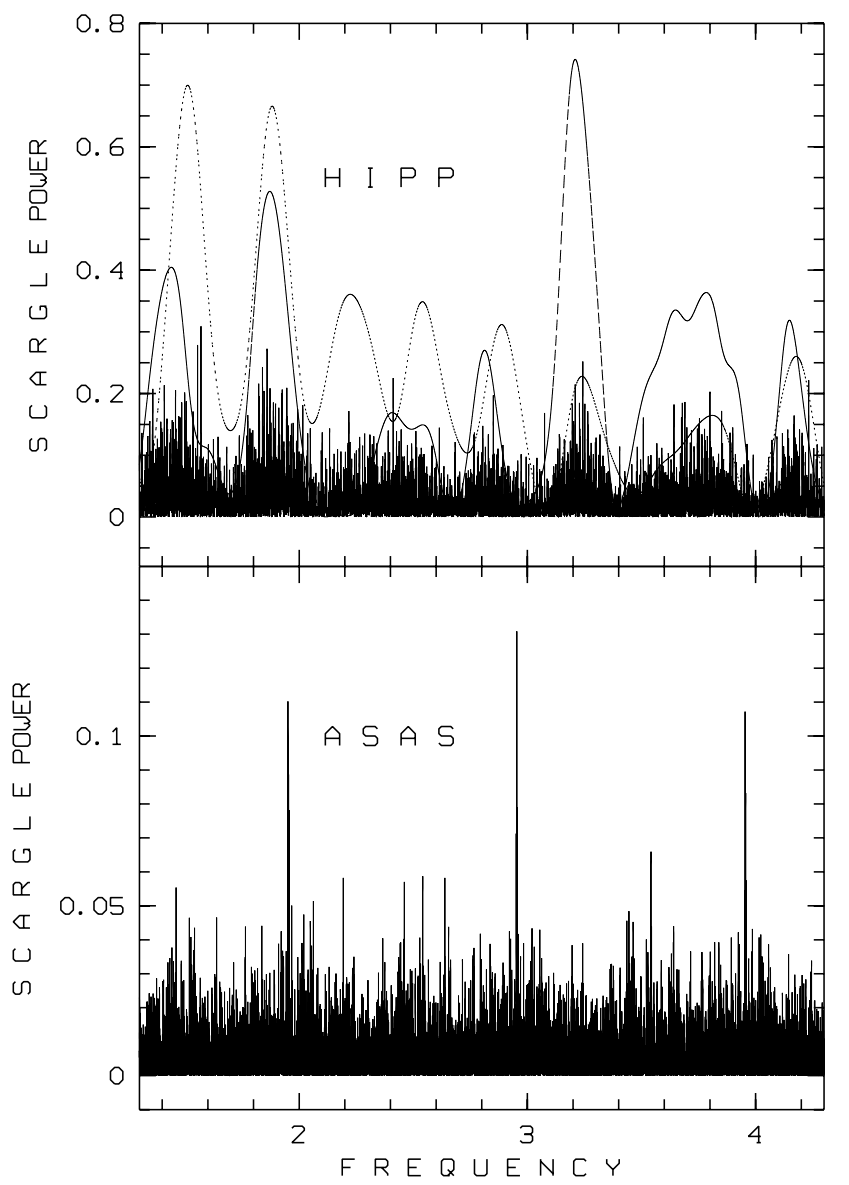

Fig. 11. Scargle power diagrams for HD 116794 . The HIPPARCos sets considered are (1) all data with uncertainties of 0.024 mag or less (full line); (2) the subset centered on HJD 2448765.1 (dashed line); and (3) the one centered on HJD 2448220.6 (dotted line).

HIPPARCos subsets are quite small. Figure 14 shows tight periodicity in 1991 and 1992, while earlier data may be contaminated by larger-amplitude irregular variations.

Assuming that $v \sin i$ lies in the range $120-150 \mathrm{~km} \mathrm{~s}^{-1}$ (see references above) the cyclic variability translates to $R \sin i$ values of 1.6-2.1 solar radii if the cycle length reflects the rotation velocity.

\subsection{Discussion}

As can be seen from Table 2, a much larger fraction of stars with spectral types corresponding to pre-ZAMS (earlier than A3, F2 and F4 for US, UCL and LCC, respectively, and luminosity class not higher than III/IV, cfr. Pecaut et al. 2012) is found to be variable than is the case for those which are firmly on the main sequence. Less than five percent of the latter show detectable variability in the ASAS $V$-band, while the rate is nearly one quarter for the pre-ZAMS stars with a slight tendency of increase towards later spectral types. The pre-ZAMS stars show mainly irregular light variations, often with short-cyclic variations of the BY Dra type superimposed.

The cycle length of the presumed spotted variables in the Sco-Cen complex correlates well with their apparent rotation velocities as measured by Chen et al. (2011), giving support to the hypothesis of rotational variability. Statistically, one expects half of the $R \sin i$ values to be between $0.866 R$ and $R$, and only $13 \%$ to
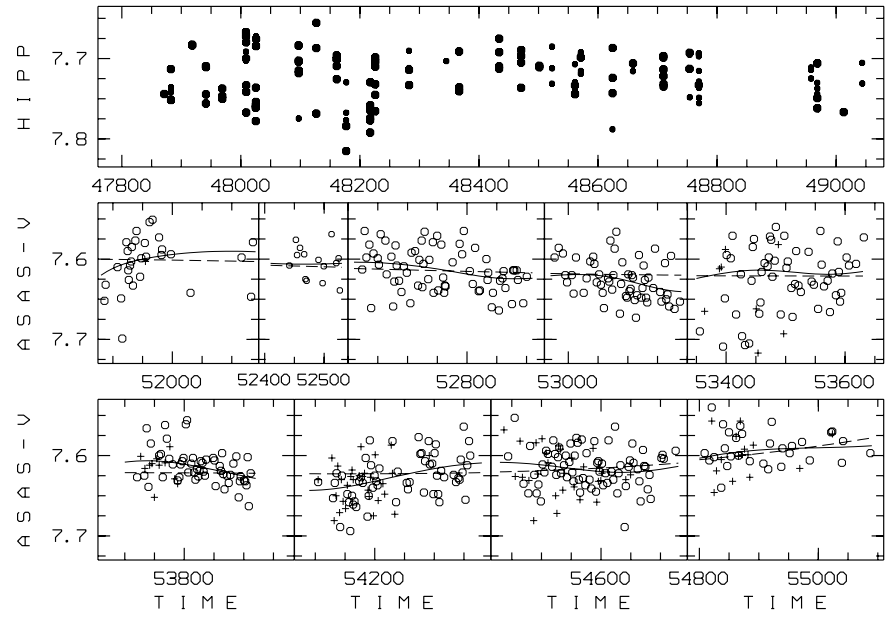

Fig. 12. Aperture-3 ASAS and HIPPARCos photometry of HD 119022. Larger symbols in the upper panels correspond to the average of two HIPPARCOS mesurements within 20 minutes. In the other panels, ASAS subsets are represented by "o" or "+" (shifted to 0.014 mag fainter magnitudes. Dashed and full lines more and less conservative long-term spline de-trendings applied to test for short-term periodicities.

be below $0.5 R$. Despite the fact that a certain spread in radii is expected for these F- and G-type pre-main sequence stars, Fig. 15 suggests that the majority of these spotted stars has a radius near to two solar radii.

Checking the literature for Sco-Cen members brighter than magnitude 7, not in our ASAS sample, reveals that the mainsequence massive-star fraction of variability is over 40\%: the list includes 9 variable emission-line stars (B2 to B9); in addition, 25 B1-B3 stars of which 12 are identified as $\beta$ Cep variables or candidate $\beta$ Cep variables; 32 B5 to early-A stars of which 17 are identified as rotational variables (chemically peculiar atmospheres, magnetic fields); and 10 B-type stars showing light variations induced by binarity ( 6 eclipsing, 4 ellipsoidal variability). The total bright-star sample contains 173 stars, among which 3 are pre-ZAMS (all of them variable) and 17 are probably evolved objects ( 7 of them variable). The much lower fraction of variability among the MS members (all in the range late B to early F) in our ASAS sample is presumably due to a lack of sensitivity (noise level and broad $V$ filter, while for example magnetic A-type stars often have higher photometric amplitudes in smaller passbands and in the blue and UV spectral regions).

\section{Conclusions}

We exploited the ASAS-V database to search for light variability in the subgroups LCC, UCL and US of the Scorpius-Centaurus complex. The HIPPARCos photometry, with very different sampling strategy, was used as an additional constraint. It also significanty extends the time baseline for long-term variables. In order to pre-select candidate variables, the old ASAS database was used, as it shows a higher consistency level than the new one. We guess that the use of the Tycho catalogue (photometrically of low quality) for zero-point determination in the fields, combined with significant offsets in centering the fields from day to day, might have induced a loss of consistency.

Nevertheless, for period-search purposes and long-term variations, the new catalogue is essential, owing to its nine-year time baseline. Only grade-A observations with a minimum level of consistency between different apertures were used. On average, $12 \%$ of the grade-A data were rejected, including many obvious 


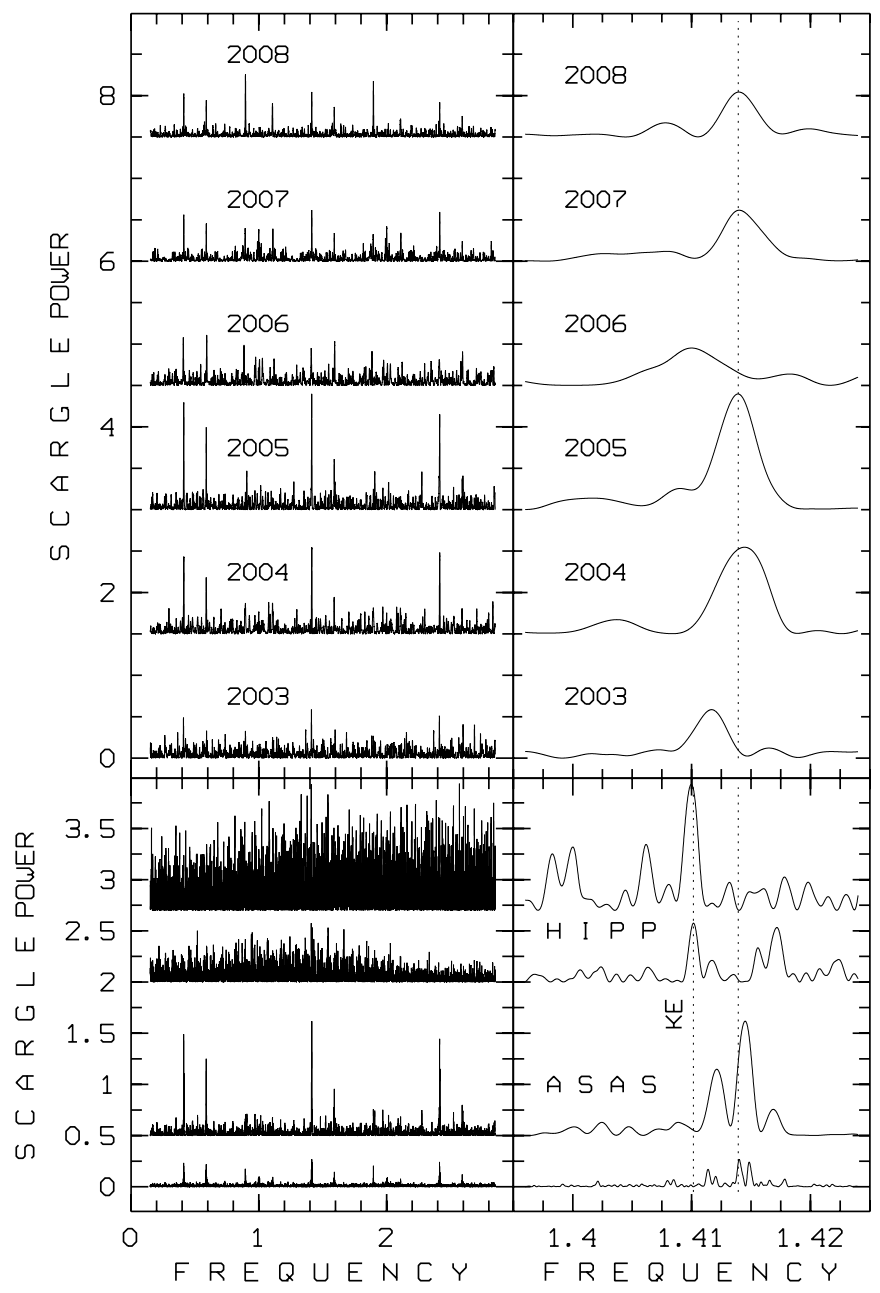

Fig. 13. Scargle power diagrams for HD 119022. The lower panels show from bottom to top: the power in the whole (2001-2009) selected ASAS set, in the one restricted to all years except 2003, 2006 and 2009, in the complete HIPPARCos data set, and in the 1991-1992 data with uncertainties of $0.013 \mathrm{mag}$ and less, respectively. A long-term trend, shown as a full line in Fig. 12 was removed from the ASAS data. The upper panels show the power in the yearly ASAS data for any year with more than 50 data points. The dotted vertical lines in the zoom panels at the right-hand side indicate the frequency found by Koen \& Eyer (2002), labeled "KE" and the frequency detected in the ASAS data from 2005.

outliers. The accepted sample sizes per object vary from 350 to 1050. Nearly two thirds of the pre-selected stars were confirmed to be light variables; a significant fraction of stars brighter than magnitude 8 turned out to have been pre-selected because the procedure was not completely insensitive to the effects of saturation in the images.

Among the 37 variables, 14 were previously unknown as such and several others had been classified only as suspected variables. In addition, for several known variables new characteristics were derived (cyclic components, additional periodicities) or known ones were improved.

Figure 16 shows the distribution of the ranges of variability for the periodic, cyclic and irregular components. It shows that periodic light variability with a total range of 0.02 mag could be identified up to $V=9.5$, and with a total range of $0.01-0.015$ mag for stars brighter than eighth magnitude. The detection level of variability amplitudes increases moderately over the range $7<V<10$. For irregular variations, the detection limit lies at

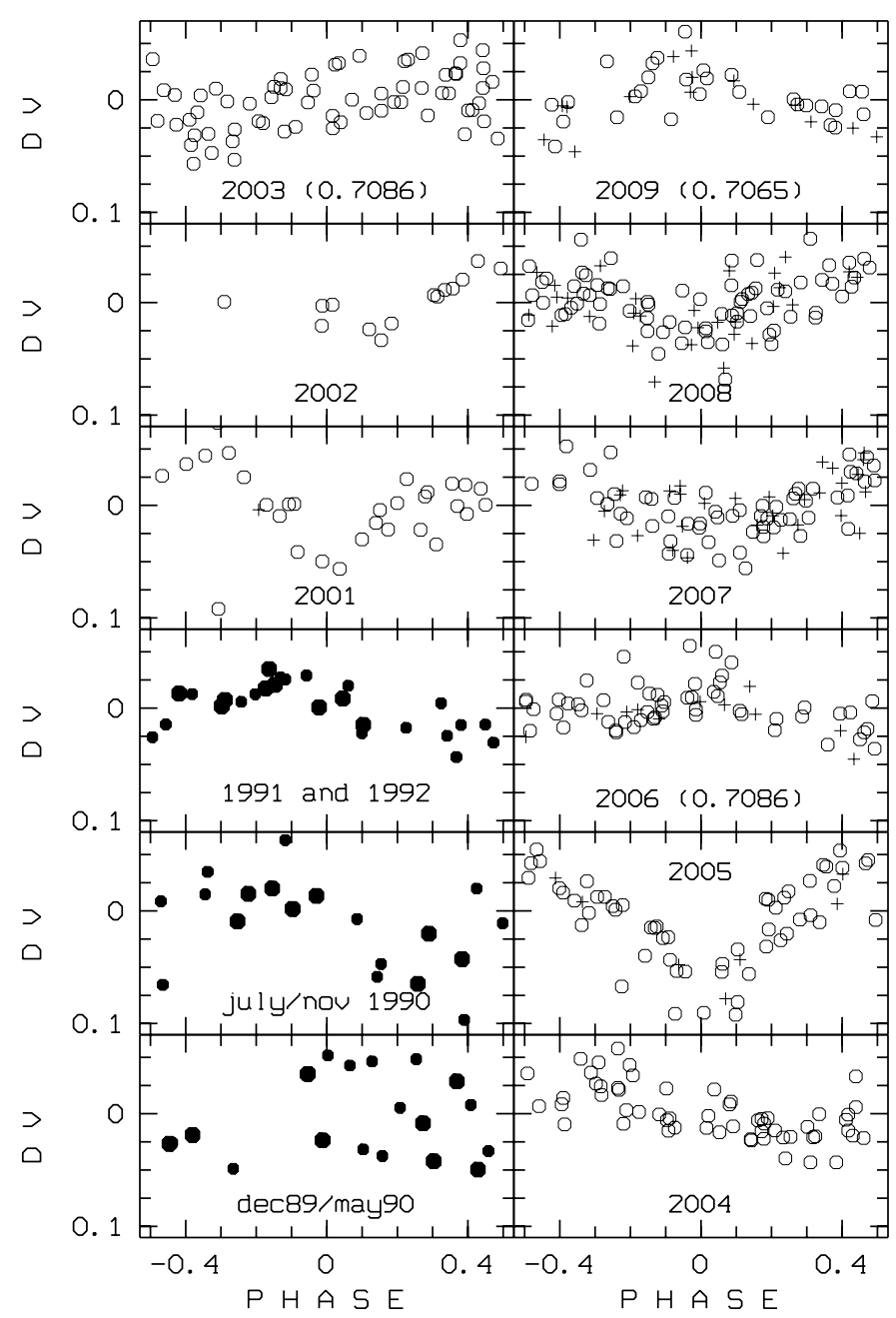

Fig. 14. Phase diagrams for HD 119022, using $P=0$ d.7092 for the HIPPARCOS data (left, lower three panels; larger symbols indicate an average of two measurements within $20 \mathrm{~min}$ ) and $P=0.7073$ for the ASAS data unless otherwise indicated between brackets in the panel. All zeropoints in phase are relative to HJD 2453600.0.

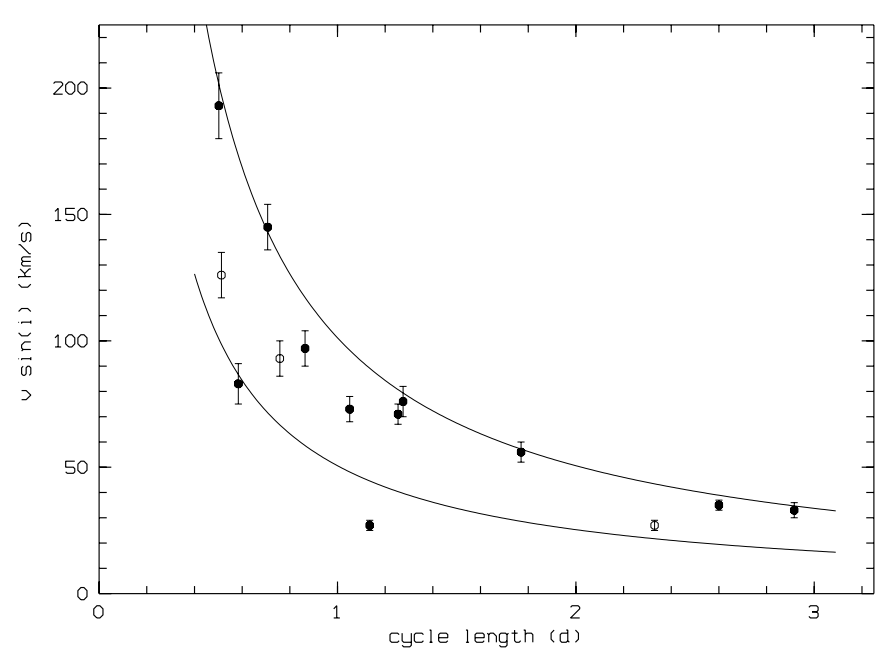

Fig. 15. Cycle length and apparent rotation velocity for presumed spotted variables in the ScoCen complex. Open symbols refer to stars with some doubt on the cycle length due to aliases or weak evidence for the reality of the cycle. Curves correspond to $R \sin i$ values of one (lower curve) and two (upper curve) solar radii. 


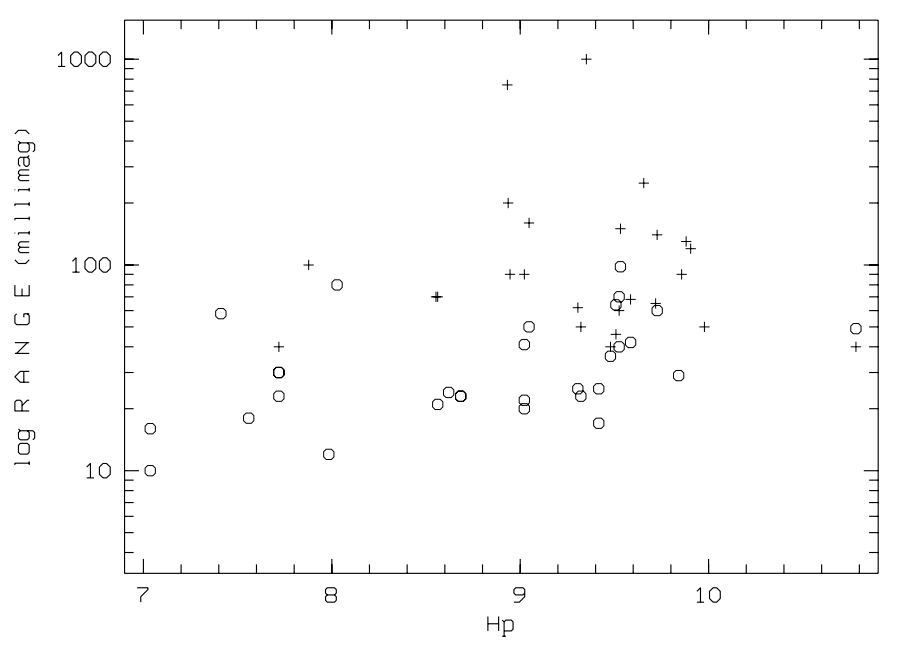

Fig. 16. Statistics of the range of variability for periodic and cyclic components ("o") and irregular or long-term variability ("+") identified in our project.

0.04 mag, taking into account that slight offsets from year to year could have an instrumental origin. For this reason we classified three late-F/early-G stars with indications of long-term variability with ranges of 0.027 to $0.035 \mathrm{mag}$, as suspected variables in Table 4.

The density of small variability ranges shows that the identification of light variables profits enormously from a relatively small gain in precision. Half of the periodic/cyclic components vary over not more than $0.025 \mathrm{mag}$. We emphasize that the present results were obtained merely by attempting to select the most consistent observations, rather than to reconsider data reduction issues, indicating that further progress should be possible. A further small gain in data reduction precision can lead to the identification of many new variables.

Another aspect that could improve the unique determination of periodicities is related to the sampling strategy which is far from ideal in the ASAS project and often provokes serious alias problems. Better strategies are known (e.g. Saunders et al. 2006). In our case, the availability of the HIPPARCos data often resolved the ambiguities present in the ASAS data sets, but this expedient is for example not feasible for fainter stars.

Acknowledgements. Part of this work was supported by the European ESA/PRODEX project number 14732/00NL/SFe(IC). The authors acknowledge extensive use of ESO-MIDAS, the Simbad and Vizier databases at CDS, Strasbourg, France and the SAO/NASA ADS services at Harvard-Smithsonian CfA, US.

\section{References}

Chen, C. H., Mamajek, E. E., Bitner, M. A., et al. 2011, ApJ, 738, 122 De Cat, P. 2007, Comm. Asteroseismol., 150, 167

de Zeeuw, P. T., Hoogerwerf, R., de Bruijne, J. H. J., Brown, A. G. A., \& Blaauw, A. 1999, AJ, 117, 354

Henry, T. J., Soderblom, D. R., Donahue, R. A., \& Baliunas, S. L. 1996, AJ, 111, 439

Hensberge, H., Vrancken, M., \& Verschueren, W. 1998, A\&A, 339, 141

Houk, N., \& Smith-Moore, M. 1988, in Michigan Catalogue of Two-dimensional Spectral Types for the HD Stars, Declinations $-26^{\circ}$ to $-12^{\circ}$, ed. W. H. Warren Jr., 4

Kiraga, M., \& Stepien, K. 2007, Acta Astron., 57, 149

Koen, C., \& Eyer, L. 2002, MNRAS, 331, 45

Levato, H., Malaroda, S., Morrell, N., \& Solivella, G. 1987, ApJS, 64, 487

Maitzen, H. M., Albrecht, R., \& Heck, A. 1978, A\&A, 62, 199

Mamajek, E. E., Meyer, M. R., \& Liebert, J. 2002, AJ, 124, 1670

Mason, B. D., Henry, T. J., Hartkopf, W. I., ten Brummelaar, T., \& Soderblom, D. R. 1998, AJ, 116, 2975

Ogura, K., \& Ishida, K. 1981, PASJ, 33, 149

Pecaut, M. J., Mamajek, E. E., \& Bubar, E. J. 2012, ApJ, 746, 154

Pigulski, A., \& Pojmański, G. 2008, A\&A, 477, 917

Pojmański, G. 2001, in IAU Colloq. 183, Small Telescope Astronomy on Global Scales, eds. B. Paczynski, W.-P. Chen, \& C. Lemme, ASP Conf. Ser., 246, 53 Pojmański, G. 2002, Acta Astron., 52, 397

Pojmański, G., \& Maciejewski, G. 2004, Acta Astron., 54, 153

Saunders, E. S., Naylor, T., \& Allan, A. 2006, A\&A, 455, 757

Soderblom, D. R., King, J. R., \& Henry, T. J. 1998, AJ, 116, 396

Torres, C. A. O., Quast, G. R., da Silva, L., et al. 2006, A\&A, 460, 695

Xing, L. F. 2010, ApJ, 723, 1542 